\title{
ON A SEMILINEAR ELLIPTIC NEUMANN PROBLEM WITH ASYMMETRIC NONLINEARITIES
}

\author{
J.-P. GOSSEZ AND P. OMARI
}

\begin{abstract}
We consider the Neumann problem (1.1) below. We extend the range of applicability of the sharp nonresonance condition derived in [Go-Om] so as, in particular, allow asymmetric nonlinearities.
\end{abstract}

\section{INTRODUCTION}

This paper is mainly concerned with the Neumann problem

$$
\left\{\begin{array}{l}
-\Delta u=g(u)-h(x) \text { in } \Omega \\
\partial u / \partial \nu=0 \text { on } \partial \Omega
\end{array}\right.
$$

Here $\Delta$ is the Laplacian operator, $\Omega$ is a bounded domain of $\mathbb{R}^{N}, N \geq 1$, with $C^{1,1}$ boundary and unit exterior normal $\nu, g$ is a continuous function on $\mathbb{R}$ and $h$ is taken in $L^{\infty}(\Omega)$ (or in $L^{p}(\Omega)$ ).

In a previous work [Go-Om], we showed that if

$$
\limsup _{s \rightarrow \pm \infty} \frac{g(s)}{s} \leq \lambda_{2} \text { and } \underset{\substack{s \rightarrow+\infty \\(\text { or } s \rightarrow-\infty)}}{\limsup _{s \rightarrow-\infty}} \frac{2 G(s)}{s^{2}}<\lambda_{2},
$$

then a necessary and sufficient condition for (1.1) to be solvable for any $h \in$ $L^{\infty}(\Omega)$ is that the range of $g$ be all of $\mathbb{R}$. Here $\lambda_{1}=0<\lambda_{2}<\cdots$ denote the distinct eigenvalues of $-\Delta$ on $\Omega$ with Neumann boundary condition, and $G(s)=\int_{0}^{s} g(t) d t$. The surjectivity of $g$ thus provides a nonresonance condition at the eigenvalue $\lambda_{1}=0$. We refer to [Go-Om] for historical background and detailed bibliography.

Condition (1.2) controls the possible interference of the nonlinearity $g$ with the higher part of the spectrum of the associated linear operator. That a restriction of this sort is needed follows trivially by considering the linear example $g(s)=\lambda_{k} s, k \geq 2$. Our purpose in the present paper is to weaken this condition (1.2) in light of two recent contributions in the area.

The first of these contributions concerns the Dirichlet problem

$$
\left\{\begin{array}{l}
-\Delta u=g(u)-h(x) \text { in } \Omega, \\
u=0 \text { on } \partial \Omega
\end{array}\right.
$$

Received by the editors June 22, 1993; originally communicated to the Proceedings of the AMS by Barbara L. Keyfitz.

1991 Mathematics Subject Classification. Primary 35J65, 35J25.

This work was partially supported by EC grant CHRX-CT94-0555.

(C)1995 American Mathematical Society 
An improvement of the interesting result of [Co-Ol] was given in [DS-Om] in the case of autonomous nonlinearities (as considered here). It amounts e.g. to replacing a restriction from above on $\lim \sup 2 G(s) / s^{2}$ by exactly the same restriction from above on $\lim \inf 2 G(s) / s^{2}$. The second contribution concerns the Fučik spectrum for $-\Delta$ with, say, Neumann boundary condition. This is defined as the set $\Sigma$ of those $\left(\lambda_{+}, \lambda_{-}\right) \in \mathbb{R}^{2}$ such that

$$
\left\{\begin{array}{l}
-\Delta u=\lambda_{+} u^{+}-\lambda_{-} u^{-} \quad \text { in } \Omega, \\
\partial u / \partial \nu=0 \text { on } \partial \Omega
\end{array}\right.
$$

admits a nontrivial solution. Clearly $\Sigma$ contains the two lines $\mathbb{R} \times\{0\}$ and $\{0\} \times \mathbb{R}$. It was shown in [DF-Go] that $\Sigma$ contains a first (nontrivial) curve $C_{1}$, which passes through $\left(\lambda_{2}, \lambda_{2}\right)$, is continuous, strictly decreasing, symmetric with respect to the diagonal and, for $N \geq 2$, asymptotic to the above two lines. This of course paves the way to considering asymmetric nonlinearities in problem (1.1).

Theorem 1.1. Assume that, for some $\left(\lambda_{+}, \lambda_{-}\right) \in C_{1}$,

$$
\limsup _{s \rightarrow-\infty} \frac{g(s)}{s} \leq \lambda_{-} \text {and } \limsup _{s \rightarrow+\infty} \frac{g(s)}{s} \leq \lambda_{+} \text {, }
$$

with moreover

$$
\liminf _{s \rightarrow-\infty} \frac{2 G(s)}{s^{2}}<\lambda_{-} \text {or } \liminf _{s \rightarrow+\infty} \frac{2 G(s)}{s^{2}}<\lambda_{+} .
$$

Then a necessary and sufficient condition for (1.1) to be solvable for any $h \in$ $L^{\infty}(\Omega)$ is that the range of $g$ be all of $\mathbb{R}$.

The proof of Theorem 1.1 is given in $\S 2$. We observe in this respect that one of the main tricks in [DS-Om] consists in deriving an estimate on a line integral from an estimate on a volume integral. This line should connect points in $\bar{\Omega}$ where the solutions are suitably controlled. In the case of the Dirichlet problem (1.3), one extremity can be taken on $\partial \Omega$, due to the boundary condition. Adapting this idea to the Neumann boundary condition requires some care. The technical details are contained in the rather lengthy Claims 5 and 6 of $\S 2$. We also observe that introducing asymmetric nonlinearities in the approach of [DS-Om] leads to another difficulty, at the level of Lemma 3.1 of [DS-Om], whose proof has a definite linear character. Claim 2 of $\S 2$, which uses some observations from [H-O-Z], shows how to bypass this lemma, by deriving directly one of its crucial consequences (i.e. (2.10) below).

In $\S 3$ we briefly consider two other situations where the techniques developed in $\S 2$ can be applied.

\section{Proof of Theorem 1.1}

The necessary part is easily derived, as in [Go-Om]. The sufficient part is a direct consequence of the following

Proposition 2.1. Assume (1.5) and (1.6), and let $h \in L^{\infty}(\Omega)$. Then (1.1) is solvable if 
Proof of Proposition 2.1. Let $A$ and $B$ be constants such that

$$
g(A)=\operatorname{essinf} h(x) \text { and } g(B)=\operatorname{ess} \sup h(x) .
$$

Clearly $A$ is an upper solution and $B$ is a lower solution for (1.1). Consequently, if $A \geq B$, the conclusion follows from the standard method of upperlower solutions. Therefore let us assume from now on $A<B$. We can further assume that

$$
\limsup _{s \rightarrow-\infty} g(s)<+\infty \text { and } \liminf _{s \rightarrow+\infty} g(s)>-\infty \text {. }
$$

Indeed otherwise there would exist either a constant lower solution $B^{\prime}<A$ or a constant upper solution $A^{\prime}>B$, and in any case one could conclude as above. We can further assume that

$$
A<0<B .
$$

Indeed otherwise one could reduce to this situation by the change of variable $v=u-(A+B) / 2$.

We will use the following homotopy:

$$
\left\{\begin{array}{l}
-\Delta u=\mu[g(u)-h(x)]+(1-\mu) \eta u \text { in } \Omega, \\
\partial u / \partial \nu=0 \text { on } \partial \Omega,
\end{array}\right.
$$

where $\eta$ is fixed with $0<\eta<\min \left\{\lambda_{+}, \lambda_{-}\right\}$and $\mu$ varies in $[0,1]$. Let us fix $p>N$. We observe that since $g$ has linear growth (a consequence of $(1.5)$ and (2.3)) and $h \in L^{\infty}(\Omega)$, any solution $u \in H^{1}(\Omega)$ of (2.5) belongs to $W^{2, p}(\Omega)$, and therefore the equation in (2.5) is satisfied a.e. in $\Omega$ and $u \in C^{1}(\bar{\Omega})$.

Our proof consists in building in $C^{0}(\bar{\Omega})$ an open bounded set $\mathscr{O}$, with $0 \in$ $\mathscr{O}$, such that no solution of $(2.5)$ with $\mu \in[0,1[$ occurs on the boundary $\partial \mathscr{O}$. Homotopy invariance of the degree then yields the conclusion of Proposition 2.1. This open set $\mathscr{O}$ will have the form

$$
\mathscr{O}=\left\{u \in C^{0}(\bar{\Omega}) ; R<\min u<B \quad \text { and } A<\max u<S\right\},
$$

where $A$ and $B$ are as above and where $R$ and $S$ will be chosen after Claim 5 below.

Claim 1. If $u$ is a solution of $(2.5)$ for some $\mu \in[0,1[$, then

$$
\max u \neq A \text { and } \min u \neq B \text {. }
$$

Proof of Claim 1. Assuming that e.g. $\max u=A$, one derives a contradiction exactly as on p. 439 of [Go-Om], by using the interior and the boundary forms of the strong maximum principle in Sobolev spaces.

The following Claims 2, 3 and 5 give properties of solutions $u$ of (2.5) for $\mu \in[0,1]$ which satisfy

$$
A \leq u(x) \leq B \quad \text { for at least one } x \in \bar{\Omega}
$$

(here $A$ and $B$ can in fact be arbitrary fixed numbers).

Claim 2. Let $\left(u_{n}, \mu_{n}\right)$ be a sequence of solutions of (2.5), satisfying (2.8) and such that $\left\|u_{n}\right\|_{\infty} \rightarrow+\infty$. Then, for a subsequence, $u_{n} /\left\|u_{n}\right\|_{\infty}$ converges weakly in $W^{2, p}(\Omega)$ to some function $v \not \equiv 0$ such that

$$
\left\{\begin{array}{l}
-\Delta v=\lambda_{+} v^{+}-\lambda_{-} v^{-} \text {in } \Omega \\
\partial v / \partial \nu=0 \text { on } \partial \Omega
\end{array}\right.
$$


$\mu_{n} \rightarrow 1$ and

$$
\left\|u_{n}\right\|_{\infty}^{-1} \int_{\Omega}\left|g\left(u_{x}\right)-\left(\lambda_{+} u_{n}^{+}-\lambda_{-} u_{n}^{-}\right)\right| d x \rightarrow 0
$$

Proof of Claim 2. First, using (1.5) and (2.3), we can write, as in Proposition 2.1 of [H-O-Z], $g(s)=p(s) s^{+}-q(s) s^{-}+r(s)$, with $p, q, r$ continuous functions such that

$$
\begin{gathered}
0 \leq p(s) \leq \lambda_{+}, \quad 0 \leq q(s) \leq \lambda_{-} \quad \text { for all } s \in \mathbb{R} \\
r(s) / s \rightarrow 0 \text { as }|s| \rightarrow+\infty
\end{gathered}
$$

Let us put $v_{n}=u_{n} /\left\|u_{n}\right\|_{\infty}$. Then $v_{n}$ satisfies

$$
\left\{\begin{array}{c}
-\Delta v_{n}=\left(\mu_{n} p\left(u_{n}\right)+\left(1-\mu_{n}\right) \eta\right) v_{n}^{+}-\left(\mu_{n} q\left(u_{n}\right)+\left(1-\mu_{n}\right) \eta\right) v_{n}^{-} \\
\quad+\mu_{n}\left(r\left(u_{n}\right)-h(x)\right) /\left\|u_{n}\right\|_{\infty} \text { in } \Omega \\
\partial v_{n} / \partial \nu=0 \text { on } \partial \Omega .
\end{array}\right.
$$

This implies that $v_{n}$ remains bounded in $W^{2, p}(\Omega)$ and so, for a subsequence, $v_{n} \rightarrow v$ weakly in $W^{2, p}(\Omega)$ where $\|v\|_{\infty}=1$. Moreover, by (2.8), $v$ vanishes somewhere in $\bar{\Omega}$. We can also suppose that for a further subsequence, $\mu_{n} \rightarrow$ $\mu \in[0,1]$ and $\mu_{n} p\left(u_{n}\right)+\left(1-\mu_{n}\right) \eta$ and $\mu_{n} q\left(u_{n}\right)+\left(1-\mu_{n}\right) \eta$ converge in $L^{\infty}(\Omega)$, with respect to the weak* topology, to some $p_{0}, q_{0} \in L^{\infty}(\Omega)$ respectively. By (2.11) these functions satisfy $0 \leq p_{0}(x) \leq \lambda_{+}$and $0 \leq q_{0}(x) \leq \lambda_{-}$a.e. in $\Omega$, and by (2.12), $\mu_{n}\left(r\left(u_{n}\right)-h(x)\right) /\left\|u_{n}\right\|_{\infty} \rightarrow 0$ uniformly a.e. in $\Omega$. Therefore $v$ satisfies

$$
\left\{\begin{array}{l}
-\Delta v=p_{0}(x) v^{+}-q_{0}(x) v^{-} \text {in } \Omega \\
\partial v / \partial \nu=0 \text { on } \partial \Omega
\end{array}\right.
$$

We also observe that as long as $p_{0}$ and $q_{0}$ are considered only on the sets $\{v \geq 0\}$ and $\{v \leq 0\}$ respectively, we can assume

$$
\begin{array}{ll}
p_{0}(x)=\eta & \text { a.e. in }\{v<0\} \\
q_{0}(x)=\eta & \text { a.e. in }\{v>0\}
\end{array}
$$

We now distinguish three cases: (i) $p_{0}(x)=0$ or $q_{0}(x)=0$ a.e. in $\Omega$, (ii) $p_{0}(x)>0$ on a subset of $\Omega$ of positive measure, $q_{0}(x)>0$ on a subset of $\Omega$ of positive measure, and meas $\left\{x \in \Omega ; p_{0}(x)<\lambda_{+}\right.$and $\left.q_{0}(x)<\lambda_{-}\right\}=0$, (iii) $p_{0}(x)>0$ on a subset of $\Omega$ of positive measure, $q_{0}(x)>0$ on a subset of $\Omega$ of positive measure, and meas $\left\{x \in \Omega ; p_{0}(x)<\lambda_{+}\right.$and $\left.q_{0}(x)<\lambda_{-}\right\}>0$.

In case (i), if e.g. $p_{0}(x)=0$ a.e. in $\Omega$, then, by $(2.15), v(x) \geq 0$ on $\Omega$ and hence, by (2.14), $v$ is constant. Since $v$ vanishes somewhere, $v \equiv 0$, a contradiction. In case (iii) one also gets a contradiction from (2.14) by using Lemma 8 from [DF-Go]. In the remaining case (ii), we first observe that $v$ cannot be of one sign. Indeed if for instance $v \geq 0$ in $\Omega$, then by (2.14), $\int_{\Omega} p_{0} v=0$ and so $v$ vanishes on a set of positive measure. But this is not possible since the solutions of (2.14) enjoy the unique continuation property. 
Therefore $v$ changes sign on $\Omega$ and we have

$$
p_{0}(x)=\lambda_{+} \text {a.e. in }\{v>0\} \quad \text { and } q_{0}(x)=\lambda_{-} \text {a.e. in }\{v<0\} \text {. }
$$

Indeed, otherwise, by $(2.15)$, we should get

$$
\operatorname{meas}\left\{x \in \Omega ; p_{0}(x)<\lambda_{+} \text {and } q_{0}(x)<\lambda_{-}\right\}>0,
$$

which is a contradiction in the case we are presently considering. It follows from (2.16) that (2.14) actually reads

$$
\left\{\begin{array}{l}
-\Delta v=\lambda_{+} v^{+}-\lambda_{-} v^{-} \text {in } \Omega, \\
\partial v / \partial \nu=0 \text { on } \partial \Omega
\end{array}\right.
$$

i.e. $v$ is a generalized eigenfunction associated to $\left(\lambda_{+}, \lambda_{-}\right)$. We also deduce from (2.16) that $\mu=1$. Therefore $p\left(u_{n}\right)$ and $q\left(u_{n}\right)$ converge with respect to the weak* topology of $L^{\infty}(v>0)$ and $L^{\infty}(v<0)$ to $p_{0}$ and $q_{0}$ respectively. Hence we have

$$
\begin{aligned}
& \int_{v>0}\left|\lambda_{+}-p\left(u_{n}\right)\right| d x=\int_{v>0}\left|p_{0}-p\left(u_{n}\right)\right| d x=\int_{v>0}\left(p_{0}-p\left(u_{n}\right)\right) d x \rightarrow 0, \\
& \int_{v<0}\left|\lambda_{-}-q\left(u_{n}\right)\right| d x=\int_{v<0}\left|q_{0}-q\left(u_{n}\right)\right| d x=\int_{v<0}\left(q_{0}-q\left(u_{n}\right)\right) d x \rightarrow 0,
\end{aligned}
$$

and we deduce

$$
\begin{aligned}
\int_{\Omega}\left|g\left(u_{n}\right) /\left\|u_{n}\right\|_{\infty}-\left(\lambda_{+} v_{n}^{+}-\lambda_{-} v_{n}^{-}\right)\right| d x \\
=\int_{\Omega}\left|\left(p\left(u_{n}\right)-\lambda_{+}\right) v_{n}^{+}-\left(q\left(u_{n}\right)-\lambda_{-}\right) v_{n}^{-}+r\left(u_{n}\right) /\left\|u_{n}\right\|_{\infty}\right| d x \\
\leq \int_{\Omega}\left|p\left(u_{n}\right)-\lambda_{+}\right| v^{+} d x+\int_{\Omega}\left|q\left(u_{n}\right)-\lambda_{-}\right| v^{-} d x \\
\quad+\int_{\Omega}\left|p\left(u_{n}\right)-\lambda_{+}\right|\left|v_{n}^{+}-v^{+}\right| d x+\int_{\Omega}\left|q\left(u_{n}\right)-\lambda_{-}\right|\left|v_{n}^{-}-v^{-}\right| d x \\
\quad+\int_{\Omega}\left|r\left(u_{n}\right)\right| /\left\|u_{n}\right\|_{\infty} d x \rightarrow 0
\end{aligned}
$$

as $n \rightarrow \infty$. This concludes the proof of Claim 2 .

Claim 3. There exist constants $C>0$ and $0<\eta_{1}<1<\eta_{2}$ such that if $u$ is a solution of $(2.5)$ for some $\mu \in[0,1]$, satisfying $(2.8)$, with $\|u\|_{\infty} \geq C$, then $\min u \cdot \max u<0$ and $\eta_{1}<\max u /(-\min u)<\eta_{2}$.

Proof of Claim 3. The proof is similar to that of Lemma 3.2 in [DS-Om]. One uses Claim 2 and the fact that any nonzero generalized eigenfunction corresponding to $\left(\lambda_{+}, \lambda_{-}\right)$changes sign in $\Omega$.

Claim 4. There exist constants $a, b$ such that if $u$ is a solution of (2.5) for some $\mu \in[0,1]$, then $\|\nabla u\|_{\infty} \leq a\|u\|_{\infty}+b$.

Proof of Claim 4. The inequality of Claim 4 is a consequence of the $L^{p}$ regularity theory and of the fact that $g$ has linear growth. 
In the next claim we exploit assumption (1.6) on the primitive. Let us suppose for instance that

$$
\liminf _{s \rightarrow+\infty} \frac{2 G(s)}{s^{2}}<\lambda_{+}
$$

Claim 5. There exists a sequence $S_{n} \rightarrow+\infty$ such that if $u$ is a solution of (2.5) for some $\mu \in[0,1]$, satisfying (2.8), then $\max u \neq S_{n}$ for all $n$.

Proof of Claim 5. By (2.17) there exists a sequence $s_{n} \rightarrow+\infty$ such that

$$
\frac{2 G\left(s_{n}\right)}{s_{n}^{2}} \rightarrow \lambda<\lambda_{+}
$$

We will show that $S_{n}$ can be taken as a tail sequence of the sequence $s_{n}$. Indeed, suppose by contradiction that for a subsequence of $s_{n}$, still denoted by $s_{n}$, there are solutions $u_{n}$ of (2.9), satisfying (2.8), such that $\max u_{n}=s_{n}$. Since $\left\|u_{n}\right\|_{\infty} \rightarrow+\infty$, we deduce from Claim 2 that for a subsequence, $u_{n} /\left\|u_{n}\right\|_{\infty} \rightarrow v$ uniformly on $\bar{\Omega}$, where $v$ is a nonzero generalized eigenfunction associated to $\left(\lambda_{+}, \lambda_{-}\right)$, and that

$$
\left\|u_{n}\right\|_{\infty}^{-1} \int_{\Omega}\left|g\left(u_{n}\right)-\left(\lambda_{+} u_{n}^{+}-\lambda_{-} u_{n}^{-}\right)\right| d x \rightarrow 0
$$

To carry on our proof of Claim 5, we need the following technical result, whose proof is postponed a little bit.

Claim 6. For every $x_{0}, y_{0} \in \bar{\Omega}$, there exists a (simple piecewise linear) path $\gamma$, joining $x_{0}$ to $y_{0}$ and having range contained in $\Omega$, with the possible exception of the end points $x_{0}$ and $y_{0}$, such that, possibly for a subsequence,

$$
\left\|u_{n}\right\|_{\infty}^{-1} \int_{\gamma}\left|g\left(u_{n}\right)-\left(\lambda_{+} u_{n}^{+}-\lambda_{-} u_{n}^{-}\right)\right| \rightarrow 0,
$$

where $\int_{\gamma}$ denotes a line integral.

By Claim 3 we know that each $u_{n}$ changes sign on $\Omega$, provided $n$ is sufficiently large. Let us denote by $y_{n} \in \Omega$ a point where $u_{n}$ vanishes and let $x_{n} \in \bar{\Omega}$ be such that $u_{n}\left(x_{n}\right)=\max u_{n}$. We clearly can suppose that $x_{n} \rightarrow x_{0} \in \bar{\Omega}$ and $y_{n} \rightarrow y_{0} \in \bar{\Omega}$, and we have $v\left(x_{0}\right)=\max v$ and $v\left(y_{0}\right)=0$. Let $\gamma_{n}$ denote the path joining $x_{n}$ to $y_{n}$ constructed in the following way: first join $x_{n}$ to $x_{0}$ by a $C^{1}$ path $\sigma_{n}$ having range in $\bar{\Omega}$, then join $x_{0}$ to $y_{0}$ by the piecewise linear path $\gamma$ provided by Claim 6, and finally join $y_{0}$ to $y_{n}$ by a $C^{1}$ path $\tau_{n}$ having range in $\bar{\Omega}$. By the $C^{1,1}$ character of $\partial \Omega, \sigma_{n}$ and $\tau_{n}$ can be taken such that

$$
\ell\left(\sigma_{n}\right) \rightarrow 0 \text { and } \ell\left(\tau_{n}\right) \rightarrow 0
$$

where $\ell($ ) denotes the length of the corresponding path. Now, using the linear 
growth of $g$ and Claim 4, we have

$$
\begin{aligned}
\frac{\lambda_{+}}{2} s_{n}^{2}- & G\left(s_{n}\right)=\left[\frac{\lambda_{+}}{2}\left(u_{n}\left(x_{n}\right)^{+}\right)^{2}-\frac{\lambda_{-}}{2}\left(u_{n}\left(x_{n}\right)^{-}\right)^{2}-G\left(u_{n}\left(x_{n}\right)\right)\right] \\
& -\left[\frac{\lambda_{+}}{2}\left(u_{n}\left(y_{n}\right)^{+}\right)^{2}-\frac{\lambda_{-}}{2}\left(u_{n}\left(y_{n}\right)^{-}\right)^{2}-G\left(u_{n}\left(y_{n}\right)\right)\right] \\
= & \int_{0}^{1}\left[\lambda_{+} u_{n}^{+}\left(\sigma_{n}\right)-\lambda_{-} u_{n}^{-}\left(\sigma_{n}\right)-g\left(u_{n}\left(\sigma_{n}\right)\right)\right]\left\langle\nabla u_{n}\left(\sigma_{n}\right), \sigma_{n}^{\prime}\right\rangle d t \\
& +\int_{0}^{1}\left[\lambda_{+} u_{n}^{+}(\gamma)-\lambda_{-} u_{n}^{-}(\gamma)-g\left(u_{n}(\gamma)\right)\right]\left\langle\nabla u_{n}(\gamma), \gamma^{\prime}\right\rangle d t \\
& +\int_{0}^{1}\left[\lambda_{+} u_{n}^{+}\left(\tau_{n}\right)-\lambda_{-} u_{n}^{-}\left(\tau_{n}\right)-g\left(u_{n}\left(\tau_{n}\right)\right)\right]\left\langle\nabla u_{n}\left(\tau_{n}\right), \tau_{n}^{\prime}\right\rangle d t \\
\leq & \left(l\left(\sigma_{n}\right)+l\left(\tau_{n}\right)\right)\left\|g\left(u_{n}\right)-\left(\lambda_{+} u_{n}^{+}-\lambda_{-} u_{n}^{-}\right)\right\|_{\infty}\left\|\nabla u_{n}\right\|_{\infty} \\
& +\left(\int_{\gamma}\left|g\left(u_{n}\right)-\left(\lambda_{+} u_{n}^{+}-\lambda_{-} u_{n}^{-}\right)\right|\right)\left\|\nabla u_{n}\right\|_{\infty} \\
\leq & {\left[l\left(\sigma_{n}\right)+l\left(\tau_{n}\right)+\left\|u_{n}\right\|_{\infty}^{-1} \int_{\gamma}\left|g\left(u_{n}\right)-\left(\lambda_{+} u_{n}^{+}-\lambda_{-} u_{n}^{-}\right)\right|\right] } \\
& \cdot\left(a\left\|u_{n}\right\|_{\infty}+b\right) \cdot\left(c\left\|u_{n}\right\|_{\infty}+d\right),
\end{aligned}
$$

for some constants $c$ and $d$. Therefore, by Claim 6 and (2.19),

$$
\frac{\lambda_{+}}{2} s_{n}^{2}-G\left(s_{n}\right) \leq \varepsilon_{n}\left(\left\|u_{n}\right\|_{\infty}^{2}+1\right)
$$

where $\varepsilon_{n} \rightarrow 0$. Using now (2.18) and Claim 3, we get that, for $n$ sufficiently large,

$$
\frac{\lambda_{+}}{2} s_{n}^{2}-G\left(s_{n}\right) \geq \frac{1}{4}\left(\lambda_{+}-\lambda\right) s_{n}^{2} \geq \frac{1}{4}\left(\lambda_{+}-\lambda\right) \eta_{1}^{2}\left\|u_{n}\right\|_{\infty}^{2} .
$$

Combining (2.20) and (2.21) leads to a contradiction when $n \rightarrow \infty$. Hence Claim 5 is proved.

We are now ready to conclude the proof of Proposition 2.1. We will show how to choose $R$ and $S$ in the definition of an open set $\mathscr{O}$ in (2.6). Let us take $n$ such that $S_{n}>\max \{-A, B, C\}$, where $A, B$ were introduced at the beginning of the proof of Proposition 2.1 and where $C$ comes from Claim 3. Let us put $S=S_{n}$ and $R=-S / \eta_{1}$, and let us consider the corresponding open set $\mathscr{O}$. We will show that there is no solution of $(2.5)$ on $\partial \mathscr{O}$ for $\mu \in[0,1[$. If $u \in \overline{\mathscr{O}}$ (i.e. $R \leq \min u \leq B$ and $A \leq \max u \leq S$ ) is a solution of (2.5) for some $\mu \in[0,1[$, then, by Claim $1, \max u>A$ and $\min u<B$. Moreover, if $\|u\|_{\infty} \leq C$, then $\max u \leq C<S$ and $\min u \geq-C>-S / \eta_{1}=R$. On the other hand if $\|u\|_{\infty}>C$, then $\max u<S$ by Claim 5 and $\min u>-S / \eta_{1}=R$ by Claim 3. In any case $u \in \mathscr{O}$. This completes the proof of Proposition 2.1.

It remains to give the

Proof of Claim 6. We can of course suppose here $N \geq 2$. Since $\Omega$ is open and connected, for any pair of points in $\Omega$, there exists a simple piecewise linear path joining them and having range in $\Omega$. Moreover, by the $C^{1,1}$ character 
of $\partial \Omega$ (which implies the interior sphere condition at any point of $\partial \Omega$ ), the above property extends to any pair of points in $\bar{\Omega}$. Therefore let $\delta$ be a simple piecewise linear path joining $x_{0}$ to $y_{0}$ and having range in $\Omega$, with the possible exception of the endpoints $x_{0}$ and $y_{0}$. Using again the interior sphere condition in case $x_{0}$ or $y_{0}$ belongs to $\partial \Omega$, we can find, for each segment $\left[z_{k}, z_{k+1}\right]$ constituting $\delta$, a $(N-1)$-dimensional ball $B_{k}$, centered at $\left(z_{k}+z_{k+1}\right) / 2$ and lying in the hyperplane orthogonal to $\left[z_{k}, z_{k+1}\right]$ through $\left(z_{k}+z_{k+1}\right) / 2$, such that the convex hull $C_{k}$ of the set $B_{k} \cup\left\{z_{k}, z_{k+1}\right\}$ is entirely contained in $\Omega$, with the possible exception of $z_{k}$ and $z_{k+1}$. Clearly $C_{k}$ is made of two symmetric closed cones $C_{k}^{-}$and $C_{k}^{+}$having as common basis $B_{k}$, as common axis the line through $z_{k}$ and $z_{k+1}$, and as vertices $z_{k}$ and $z_{k+1}$, respectively. Let us set, for each $n$,

$$
\Gamma_{n}=\left|g\left(u_{n}\right)-\left(\lambda_{+} u_{n}^{+}-\lambda_{-} u_{n}^{-}\right)\right| /\left\|u_{n}\right\|_{\infty} .
$$

We know that $\int_{\Omega} \Gamma_{n} d x \rightarrow 0$ and therefore, for each $k$,

$$
\int_{C_{k}^{-}} \Gamma_{n} d x \rightarrow 0 \text { and } \int_{C_{k}^{+}} \Gamma_{n} d x \rightarrow 0 .
$$

Now we introduce spherical coordinates in $C_{k}^{-}$. Without loss of generality, we can suppose that $z_{k}$ is the origin of $\mathbb{R}^{N}$ and that the line through $z_{k}$ and $z_{k+1}$ is the $x_{N}$ axis. We will also assume $N \geq 3$ (the case $N=2$ can be treated in a similar way). We denote the spherical coordinates of a point $x \in C_{k}^{-}$by $\left(\rho, \varphi_{1}, \ldots, \varphi_{N-2}, \theta\right)$, with $\rho \in\left[0, R_{k} / \cos \varphi_{1}\right], \varphi_{1} \in\left[0, \alpha_{k}\right]$, $\left(\varphi_{2}, \ldots, \varphi_{N-2}, \theta\right) \in[0, \pi]^{N-3} \times[0,2 \pi]$, where $R_{k}=\frac{1}{2}\left|z_{k+1}-z_{k}\right|$ and $\alpha_{k}$ is the semi-amplitude of $C_{k}^{-}$. Hence, indicating by $\Phi$ this change of coordinates, we get

$$
\begin{aligned}
& \int_{\left[0, \alpha_{k}\right] \times[0, \pi]^{N-3} \times[0,2 \pi]}\left(\int_{0}^{R_{k} / \cos \varphi_{1}} \Gamma_{n}(\Phi)\left|\operatorname{det} \Phi^{\prime}\right| d \rho\right) d \varphi_{1} \cdots d \varphi_{N-2} d \theta \\
& =\int_{C_{k}^{-}} \Gamma_{n}(x) d x \rightarrow 0
\end{aligned}
$$

where $\left|\operatorname{det} \Phi^{\prime}\right|=\rho^{N-1}\left(\sin \varphi_{1}\right)^{N-2}\left(\sin \varphi_{2}\right)^{N-3} \cdots\left(\sin \varphi_{N-2}\right)$. It follows that, possibly for a subsequence,

$$
\int_{0}^{R_{k} / \cos \varphi_{1}} \Gamma_{n}(\Phi) \rho^{N-1} d \rho \rightarrow 0,
$$

for a.e. $\left(\varphi_{1}, \ldots, \varphi_{N-2}, \theta\right) \in\left[0, \alpha_{k}\right] \times[0, \pi]^{N-3} \times[0,2 \pi]$. Passing to a further subsequence we also have that for a.e. fixed $\left(\varphi_{1}, \ldots, \varphi_{N-2}, \theta\right), \Gamma_{n}(\Phi) \rightarrow 0$ for a.e. $\rho \in\left[0, R_{k} / \cos \varphi_{1}\right]$. On the other hand the functions $\Gamma_{n}$ are uniformly bounded (due to the linear growth of $g$ ) and therefore, by the Lebesgue dominated convergence theorem, we get that, for a.e. $\left(\varphi_{1}, \ldots, \varphi_{N-2}, \theta\right)$,

$$
\int_{0}^{R_{k} / \cos \varphi_{1}} \Gamma_{n}(\Phi) d \rho \rightarrow 0 .
$$

This means that, for a.e. point $\zeta$ in $B_{k}$,

$$
\int_{\left[z_{k}, \zeta\right]} \Gamma_{n} \rightarrow 0
$$


Working similarly on $C_{k}^{+}$, one concludes that, for a.e. $\zeta$ in $B_{k}$,

$$
\int_{\left[\zeta, z_{k+1}\right]} \Gamma_{n} \rightarrow 0
$$

Therefore, setting $\gamma_{k}=\left[z_{k}, \zeta\right] \cup\left[\zeta, z_{k+1}\right]$ for one $\zeta$ such that both (2.22) and (2.23) hold, one has

$$
\left\|u_{n}\right\|_{\infty}^{-1} \int_{\gamma_{k}}\left|g\left(u_{n}\right)-\left(\lambda_{+} u_{n}^{+}-\lambda_{-} u_{n}^{-}\right)\right| \rightarrow 0 .
$$

Repeating this argument on each $C_{k}$, one eventually gets the conclusion by taking $\gamma=\bigcup_{k} \gamma_{k}$.

Remark 2.3. The result of Theorem 1.1 easily extends to the problem

$$
\left\{\begin{array}{l}
L u=g(u)-h(x) \text { in } \Omega, \\
\partial u / \partial \nu_{L}=0 \text { on } \partial \Omega,
\end{array}\right.
$$

where $L$ is an elliptic operator of the form

$$
L u=-\sum_{i, j=1}^{N} \frac{\partial}{\partial x_{j}}\left(a_{i j}(x) \frac{\partial u}{\partial x_{i}}\right)
$$

with Lipschitz coefficients $a_{i j}=a_{j i}$ and where $\partial / \partial \nu_{L}$ denotes the associated conormal derivative. Similar extensions hold for Theorems 3.1 and 3.2 below.

\section{TWO RELATED PROBLEMS}

The techniques developed in $\S 2$ can be applied to the situation where the nonlinearity $g$ in problem $(1.1)$ is such that $g(s) / s$ lies asymptotically between two higher eigenvalues $\lambda_{k}$ and $\lambda_{k+1}$. One obtains in this way an analogue for the Neumann problem of the main results of [DS-Om].

Theorem 3.1. Assume that, for some $k>1$,

$$
\lambda_{k} \leq \liminf _{s \rightarrow \pm \infty} \frac{g(s)}{s} \leq \limsup _{s \rightarrow \pm \infty} \frac{g(s)}{s} \leq \lambda_{k+1} .
$$

Moreover assume that

$$
\limsup _{s \rightarrow-\infty} \frac{2 G(s)}{s^{2}}>\lambda_{k} \quad \text { or } \limsup _{s \rightarrow+\infty} \frac{2 G(s)}{s^{2}}>\lambda_{k}
$$

and that

$$
\liminf _{s \rightarrow-\infty} \frac{2 G(s)}{s^{2}}<\lambda_{k+1} \text { or } \liminf _{s \rightarrow-\infty} \frac{2 G(s)}{s^{2}}<\lambda_{k+1} .
$$

Then problem (1.1) is solvable for any given $h \in L^{p}(\Omega)$ with $p>N$.

These techniques also allow one to deal with the Dirichlet problem (1.3) when the nonlinearity $g$ is such that $g(s) / s$ lies asymptotically between the first eigenvalue and one point of the first curve of the corresponding Fučik spectrum. 
Theorem 3.2. Let $\mu_{1}$ be the first eigenvalue of $-\Delta$ on $H_{0}^{1}(\Omega)$ and let $\left(\mu_{+}, \mu_{-}\right)$ be one point of the first curve of the Fučik spectrum of $-\Delta$ on $H_{0}^{1}(\Omega)$. Assume that

$$
\mu_{1} \leq \liminf _{s \rightarrow \pm \infty} \frac{g(s)}{s} \leq \limsup _{s \rightarrow \pm \infty} \frac{g(s)}{s} \leq \mu_{ \pm} .
$$

Moreover assume that

$$
\limsup _{s \rightarrow-\infty} \frac{2 G(s)}{s^{2}}>\mu_{1} \text { and } \limsup _{s \rightarrow+\infty} \frac{2 G(s)}{s^{2}}>\mu_{1},
$$

and that

$$
\liminf _{s \rightarrow-\infty} \frac{2 G(s)}{s^{2}}<\mu_{-} \quad \text { or } \quad \liminf _{s \rightarrow+\infty} \frac{2 G(s)}{s^{2}}<\mu_{+} .
$$

Then problem (1.3) is solvable for any given $h \in L^{p}(\Omega)$ with $p>N$.

\section{REFERENCES}

[Co-Ol] D. Costa and A. Oliveira, Existence of solutions for a class of semilinear elliptic problems at double resonance, Bol. Soc. Brasil. Mat. 19 (1988), 21-37.

[DF-Go] D. De Figueiredo and J.-P. Gossez, On the first curve of the Fucik spectrum of an elliptic operator, Differential Integral Equations 7 (1994), 1285-1302.

[DS-Om] D. Del Santo and P. Omari, Nonresonance conditions on the potential for a semilinear elliptic problem, J. Differential Equations 108 (1994), 120-138.

[Go-Om] J.-P. Gossez and P. Omari, A necessary and sufficient condition of nonresonance for a semilinear Neumann problem, Proc. Amer. Math. Soc. 114 (1992), 433-442.

[H-O-Z] P. Habets, P. Omari, and F. Zanolin, Nonresonance conditions on the potential with respect to the Fucik spectrum for the periodic boundary value problem, Rocky Mountain J. Math. (to appear).

Département de Mathématique, Campus de la Plaine, CP 214, Université libre de BRuXelles, 1050 BruXelles, Belgium

E-mail address: ulbmathQulb.ac.be

Dipartimento di Scienze Matematische, Università di Trieste, Piazzale Europa 1, 34127 TRIESTE, ItALY

E-mail address: omari@utsax 7 .univ.trieste.it 